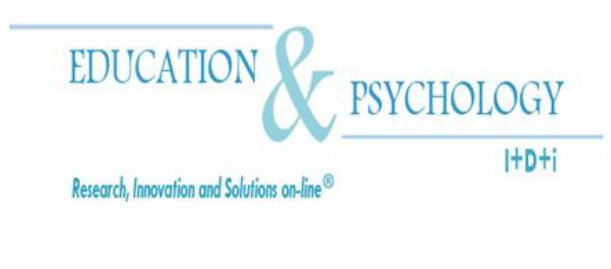

\title{
Valoración de un programa mediacional de entrenamiento de funciones cognitivas básicas para preescolares
}

\section{Sara Mata, $M^{\mathrm{a}}$ Dolores Calero, Rosario Carles}

Departamento de Personalidad, Evaluación y Tratamiento Psicológico.

Universidad de Granada

\section{España}

Correspondencia: Sara Mata. Departamento de Personalidad, Evaluación y Tratamiento Psicológico, Universidad de Granada, Campus Cartuja s/n 18071 Granada. España. E-mail: $\underline{\text { saramata@ugr.es }}$

(C) Education \& Psychology I+D+i and Editorial EOS (Spain) 


\section{Resumen}

Introducción. El estudio valora la eficacia de un programa mediacional destinado al entrenamiento cognitivo de preescolares que presentan déficits en habilidades cognitivas básicas próximas al currículo académico.

Método. La muestra estuvo compuesta por 48 preescolares en dos grupos en función de si recibían o no el programa de entrenamiento. Se ha seguido un diseño quasi-experimental prepost con grupo control equivalente.

Resultados. Los resultados muestran diferencias significativas entre el grupo tratamiento y el control tras la aplicación del programa en las funciones cognitivas entrenadas y en metacognición.

Discusión y conclusiones. Los resultados muestran la eficacia del programa al mejorar las habilidades entrenadas: clasificación y planificación verbal de los niños de Educación Infantil. Además, los efectos se transfieren a otras habilidades.

Palabras Clave: programa intervención cognitiva; preescolares, mediación; habilidades cognitivas. 


\title{
Assessment mediational training program of basic cognitive functions for preschoolers
}

\begin{abstract}
Introduction. The main objective in this study has been assessing the efficiency of a mediational intervention program for preschoolers who present deficits in basic cognitive skills, which are related with the pre-school curriculum.

Method. The sample was composed for 48 preschool children divided in two groups: the treatment group and the control group. It has used a quasi-experimental design pre-post intervention with an equivalent control group.

Results. The results show that the intervention program causes differences between the two groups in training cognitive function and metacognition.

Discussion and conclusions. The results show the program's efficiency in order to improve the trained skills: classification and verbal planification of preschool children. The effects also were transferred to other skills.
\end{abstract}

Keywords: cognitive intervention program; preschool children, mediation, cognitive skills. 


\section{Introducción}

Desde el año 1961, cuando Hunt publica su obra "Inteligencia y Experiencia", la inteligencia deja de considerarse como una entidad estable, que marca el desempeño del sujeto a lo largo de su vida, y empieza a ser considerada en interacción con ciertas variables ambientales, que modulan o influyen en ella. En los años siguientes, la importancia dada al aprendizaje, tanto a través de la exposición directa, como a través de la mediación, influye en la aparición de programas de intervención destinados a mejorar las capacidades intelectuales, el rendimiento académico y/o la capacidad para resolver problemas de sujetos con bajo rendimiento intelectual.

Inicialmente se desarrollan programas de diversa índole. Por una parte, surgen los programas educativos, considerados un medio para conseguir el máximo aprovechamiento educativo de los sujetos normales en el contexto escolar. También aparecen programas de educación compensatoria que, basados en los periodos críticos, conciben el enriquecimiento ambiental como medio de compensación del déficit intelectual de niños con problemas neurológicos o madurativos o provenientes de grupos con deprivación sociocultural; y, por último, los programas de entrenamiento cognitivo, que pretenden la modificación de la capacidad intelectual, de los procesos cognitivos y metacognitivos, de las estrategias de pensamiento y de resolución de problemas y de las habilidades de aprendizaje (Calero, 1995; Justicia, Amezcua y Pichardo, 2000).

Entre los autores de programas de entrenamiento cognitivo son de obligada mención Feuerstein, Rand, Hoffman y Miller (1980), quienes desarrollaron el Programa de Enriquecimiento Instrumental, basándose en la teoría de la Modificabilidad Cognitiva Estructural (Feuerstein, Feuerstein, Falik y Rand, 2002). Esta teoría postula que cualquier individuo es susceptible de mejorar su capacidad intelectual y aprender a aprender si participa en experiencias de aprendizaje mediado (EAM). A través de éstas, ciertos estímulos del medio ambiente son interceptados por un agente, que suele ser un padre o un mayor del grupo de pertenencia, quien los selecciona, los organiza, los reordena y/o los agrupa, en función de una meta específica (Feuerstein, Klein y Tannenbaum, 1991). En base a esta teoría, el Programa de Enriquecimiento Instrumental tiene como objetivo desarrollar, enriquecer y cristalizar los prerrequisitos de la inteligencia (Feuerstein, 1993). Este programa ha sido implementado y valorado en múltiples ocasiones encontrándose mejoras 
significativas en rendimiento académico, habilidades cognitivas, habilidades perceptivoespaciales, autoestima y habilidades no intectivas relevantes para el aprovechamiento educativo tales como autorregulación, planificación, etc... en los niños participantes (Campllonch, Domínguez, Beardo, Matías y Navarro, 1983; Calero, 1986; Narroll, Silverman y Waksman, 1982; Prieto, 1987; Rand, Feuerstein, Tannenbaum, Jesen y Hoffman, 1977; Ruíz Bolívar, 1988; Ruíz Bolívar y Castañeda, 1983). Otros programas de intervención cognitiva han demostrado resultados positivos en tests de ejecución intelectual y pruebas de aptitud, como es el Proyecto de Inteligencia Harvard (Domínguez, Mallon, Warnick, Nickenson, Sánchez y cols., 1980) o el programa CORAL (Cerrillo, 2002). Ambos han mostrado un aumento significativo del C. I. general, razonamiento abstracto, aptitud espacial, autoconcepto, lengua, matemáticas, etc. (Cerrillo, 2002, 2008).

Resultados como los comentados, sirven para mostrar cómo diferentes estrategias de intervención muestran la posibilidad de mejorar las habilidades cognitivas y metacognitivas, la capacidad de resolver problemas, el rendimiento académico, la comprensión social y/o la autoestima de niños inicialmente desfavorecidos y de diferentes edades (Francesc, Serrano y Carles, 2010; Meilán, Salgado, Arana, Carro y Jenaro, 2008; Soriano y Franco, 2010).

Dado que la mejor acción es la preventiva, lo ideal sería tratar de dotar a los alumnos que comienzan la Educación Infantil de herramientas para evitar problemas académicos futuros (Gutiérrez, 1999). Éste ha sido el objetivo de distintos programas de entrenamiento cognitivo destinados a los alumnos de Educación Infantil y de los primeros cursos de Primaria. Entre éstos podemos nombrar el Bright Start (Cognitive Curriculum for Young Children, Haywood, Brooks y Burns, 1986, 1992) destinado a la adquisición y aplicación de procesos cognitivos básicos de niños de entre 3 y 6 años. Programa ampliamente validado en distintos países tales como Bélgica (Vanden Wijngaert, 1991; Warnez, 1991), Israel (Tzuriel, Kaniel, Kaner y Haywood, 1997), EEUU, Canadá, Venezuela y Francia (Castañón, 2007; Haywood 2000; Tzuriel, Kaniel, Zeliger, Friedman y Haywood, 1998). En estos se ha comprobado su utilidad en niños con problemas sensoriales y de lenguaje, con problemas de aprendizaje, desventaja socio-cultural o inmigrantes y han mostrado efectos muy positivos en habilidades cognitivas y en tests de inteligencia.

El programa Refuerzo del desarrollo cognitivo, FLASH (Gutiérrez, 1999, 2002) pretende la prevención de dificultades de aprendizaje y el refuerzo cognitivo en niños de 3 a 8 
años. Según la valoración aportada por su autor, este programa produce, en los niños entrenados, diferencias significativas entre el pretest y el postest en la escala del programa FLASH, en las Matrices progresivas del Raven, Escala de color (Raven, 1996), en el REVERSAL (Edfeldt, 1955) y en la Batería de aptitudes para el aprendizaje, BAPAE (De la Cruz, 1982), por lo que concluye que el programa FLASH potencia el desarrollo intelectual y mejora el rendimiento curricular.

El Programa de entrenamiento cognitivo para niños pequeños (Saíz y Román, 2004) está destinado a niños de segundo ciclo de Infantil o primer ciclo de Primaria y sus objetivos son el entrenamiento en estrategias de resolución de problemas cognitivos y sociales. Los análisis realizados por las autoras muestran que la aplicación del programa produce cambios significativos en procesamiento de la información y en resoluación de problemas evaluados por la escala para la medición de las estrategias de resolución de problemas (Saíz, 1995a) y por la Batería de habilidades sociales en la solución de problemas interpersonales para Ciclo Inicial, BHSSPI (Pelechado, 1984, 1987).

El Progresint, Programa para la estimulación de habilidades de la inteligencia (Yuste y Sánchez, 2007) pretende la mejora de las habilidades intelectuales de niños en los primeros cursos de Educación Primaria. Estudios realizados por los autores muestran que los sujetos a los que se aplica el programa mejoran las puntuaciones en las Matrices progresivas del Raven, Escala de color y general (Raven, 1996). En definitiva, diferentes programas muestran resultados positivos en habilidades cognitivas o inteligencia cuando se utilizan estrategias de intervención con niños preescolares.

\section{Objetivos}

En esta dirección, el trabajo que aquí se presenta se ha dirigido a la valoración de la eficacia de un programa de entrenamiento cognitivo para preescolares, el Programa Mediacional de Enriquecimiento cognitivo para niños de Educación Infantil, PROMECO. (Calero, Mata y Carles, 2011) que presenta la novedad de trabajar funciones cognitivas relacionadas con el curriculum de Educación Infantil, a través de un enfoque mediacional trasversal, esto es, entrenando todas las funciones seleccionadas a partir de una sola tarea y en un breve formato de aplicación en pequeños grupos. Intentamos comprobar que los niños participantes pueden beneficiarse del programa, mejorando su ejecución previa en tareas de evaluación de dominio de las funciones entrenadas en el programa, -construidas por el grupo 
de investigación-, y transfiriendo la mejora a las habilidades cognitivas básicas evaluadas por la escala EHPAP, la Escala evaluación de habilidades y potencial de aprendizaje en preescolares, (Calero, Robles, Márquez y de la Osa, 2009), a un registro de metacognición, al test de inteligencia K-BIT (Kaufman y Kaufman, 1994) y al rendimiento académico evaluado por los profesores. En concreto, pretendemos:

1) Constatar que el grupo tratamiento consigue incrementos significativos del pretest al postest en las hablidades cognitivas entrenadas, valoradas por las tareas de evaluación de dominio.

2) Comprobar los efectos diferenciales entre grupo tratamiento y grupo control en las habilidades cognitivas evaluadas por la escala EHPAP en la fase post-tratamiento como consecuencia de la transferencia de efectos del programa.

3) Determinar si exiten efectos positivos diferenciales entre el grupo tratamiento y el control en las puntuaciones post-tratamiento en un registro de metacognición, en el K-BIT y en rendimiento académico.

\section{Método}

\section{Participantes}

En el estudio han participado 48 preescolares, de entre 5 y 6 años de edad (entre 65 y 76 meses $)(\mathrm{M}=70.42$, DT $=3.61)$, escolarizados en $3^{\circ}$ de Educación Infantil en colegios públicos de Granada (España). La muestra se dividió en dos grupos, uno tratamiento, que recibió el programa de intervención y uno control, que no recibió tratamiento. El grupo tratamiento estaba formado por 24 preescolares (10 niñas y 14 niños), (edad en meses: $\mathrm{M}=$ 69.29, DT = 3.48). El grupo control se compuso por 24 alumnos (10 niñas y 14 niños), (edad en meses: $\mathrm{M}=71.54$, DT $=3.45)$. Previamente a la participación en el estudio se controló que los niños no presentarán problemas psicológicos o de comportamiento y que contaran con el consentimiento informado de sus padres.

\section{Instrumentos}

\section{Instrumentos de evaluación}

Test breve de inteligencia de Kaufman, K-BIT (Kaufman y Kaufman, 1994). Es un test de "screening" que permite llegar a una rápida apreciación de la inteligencia general del niño mediante dos subtests: vocabulario y matrices. Suministra un C.I. verbal, un C.I. no 
verbal y un C.I. compuesto que resume el rendimiento total en el test. Sus coeficientes de fiabilidad varían según el rango de edad, pero en ningún caso baja del .76. Además, el C.I. compuesto muestra una correlación media de .63 con la suma de puntuaciones de procesamiento mental del K-ABC y de .75 con la del subtest de conocimientos del mismo test. El C.I. compuesto del K-BIT tiene una correlación de .80 con el C.I. global del WISC-R y de .75 con el WAIS-R. Estas correlaciones apoyan la validez de constructo del K-BIT.

Escala de evaluación de habilidades y potencial de aprendizaje en preescolares, EHPAP (Calero y cols., 2009). Es un procedimiento de evaluación de potencial de aprendizaje que mide la aplicación de procesos cognitivos en tareas típicas del currículo de Educación Infantil. Su rango de aplicación es de 3 a 6 años. Se compone de seis subtests: clasificación, memoria auditiva, memoria visual, planificación verbal, series y toma de perspectiva. El formato de aplicación es pretest-mediación-posttest, no obstante, en este trabajo sólo hemos empleado la fase pretest, como test de ejecución de las funciones cognitivas entrenadas. Las puntuaciones que ofrece son cuantitativas, no normativas. Distintos estudios han aportado datos sobre la fiabilidad, validez de constructo y validez discriminante del instrumento (Bensoussan, 2002; Calero, Carles, Mata y Navarro, 2010; Calero, Robles y García-Martín, 2010; Lidz, 2004; Lidz y Van der Aalsvoort, 2005; Malowitsky, 2001).

Tareas de evaluación de dominio de las funciones entrenadas en el programa. Para evaluar el efecto del programa el grupo de investigación elaboró una serie de tareas específicas sobre las funciones cognitivas y aptitudes entrenadas por el mismo: clasificación, evaluada a través de la realización de categorías de animales (en función del tamaño, forma de desplazamiento y número de patas); planificación verbal, evaluada mediante la descripción de las etapas de la acción de ducharse; memoria auditiva y recuerdo demorado, evaluadas mediante el recuerdo de una lista breve de palabras en dos fases, de forma inmediata y después de realizar la prueba de clasificación; vocabulario, con el reconocimiento de una lista visual de animales; autorregulación e interactividad, evaluadas mediante la valoración del evaluador en la interacción con el niño. La puntuación oscila de 0 a 80.

Registro de metacognición. Las habilidades metacognitivas se evaluaron a través del registro de la información verbal de los niños mientras realizaban una tarea, según un 
cuestionario construido por el equipo de investigación (Carles, Mata, López-Rubio, Navarro, Calero, Fernández-Parra, Vives y Márquez, 2010) teniendo en cuenta las dificultades de introspección que presentan los niños en estas edades (Monereo, 1994). El cuestionario estaba compuesto por 10 ítems basados en conductas, que según estudios previos, son demostrativas de metacognición en esas edades (Annervita y Vauras, 2006; Desoete, 2007; Garrett, Mazzocco, y Baker, 2006; Marcel, Van Hout-Walters y Afflerbach, 2006). Estos estudios han mostrado la presencia de tres componentes metacognitivos básicos: evaluación, autorregulación y planificación. Un estudio de las propiedades psicométricas del cuestionario (Carles, 2012) ha mostrado una alta consistencia interna (con una alpha de Cronback de .744) y la existencia de tres factores independientes, cada uno de los cuales es evaluado a través de los ítems del cuestionario. La varianza total explicada por esos tres factores es $59.82 \%$

Registro de rendimiento académico. Para evaluar el rendimiento académico se utilizó el método de evaluación estandarizada para preescolares en el sistema educativo español. Los profesores registraron el rendimiento académico de los niños en seis áreas curriculares: matemáticas, expresión oral/escrita, identidad y autonomía personal, medio físico y social, expresión corporal, y expresión artística. En cada área se puntuaba: 1) rendimiento bajo, 2) rendimiento medio y 3) rendimiento alto, siendo la puntuación mínima 6 y la máxima 18.

\section{Programa de intervención}

Programa mediacional de enriquecimiento cognitivo para niños de Educación Infantil, PROMECO (Calero y cols., 2011). Consta de seis sesiones de una hora y entrena en cada una de ellas las habilidades cognitivas de clasificación, planificación verbal, memoria auditiva y memoria auditiva/ recuerdo demorado, a la vez que se refuerza el vocabulario. El programa sigue una metodología mediacional y emplea materiales y ejercicios lúdicos. Cada una de las sesiones se guía a través de un tema central/objetivo La aplicación se realiza en grupo de 5-8 niños.

\section{Procedimiento}

Este estudio constituye la segunda fase de un proyecto inicial de investigación dirigido a determinar perfiles de aprendizaje y pautas de crianza en preescolares. En la primera fase del mismo se evaluó a niños preescolares de entre 4 y 5 años de edad con diferentes pruebas de habilidades cognitivas, potencial de aprendizaje, inteligencia, metacognición y rendimiento académico. Una vez completada la evaluación se observaron 
aquellas tareas en las que los niños tenían una ejecución más baja. Así se realizó una curva de rendimiento y se seleccionaron las funciones cognitivas en las que cierto número de niños presentaban una ejecución por debajo de la media, estas fueron: clasificación, planificación verbal, memoria auditiva, vocabulario, autorregulación e interactividad.

A partir de dicha información nos dispusimos a elaborar un programa de intervención cognitiva basado en el Mapa Cognitivo (Feuerstein y cols. 1980), esto es, tras realizar un análisis de tareas para cada función, se determinó el tipo de actividades que las entrenaban, se plantearon diferentes formatos de presentación y se graduaron niveles de dificultad. A su vez, se construyeron las tareas de evaluación de dominio de las funciones entrenadas en el programa, incluyendo tareas paralelas a las contenidas en el programa de intervención y se elaboró una guía para el profesional y un cuadernillo de ejercicios para padres. Del total de los niños seleccionados inicialmente, aproximadamente la mitad se prestó para participar en el programa, por lo que la otra mitad pasó a constituir el grupo control del estudio.

El programa se impartió en tres colegios de Granada (España), en tres grupos de entrenamiento realizados por una misma psicóloga. Antes y después del mismo se realizaron las evaluaciones pre y post en las que se evaluó individualmente a cada niño con las tareas de evaluación de dominio de las funciones entrenadas en el programa, el pretest de la escala EHPAP, con el registro de metacognición y el K-BIT. Al final del tercer trimestre se recogieron las puntuaciones de rendimiento académico de cada niño.

\section{Análisis de datos}

Se ha empleado un diseño quasi-experimental pre-post con grupo control equivalente. El análisis de los datos se ha realizado con el software estadístico SPSS versión 18.0. Los análisis estadísticos empleados han sido la $t$-Student y el Modelo Lineal General de medidas repetidas. La variable independiente ha sido el grupo de pertenencia, esto es, recibir o no el programa de intervención. Las variables dependientes han sido las puntuaciones obtenidas en las tareas de evaluación de dominio de las funciones entrenadas en el programa, la EHPAP, registro de metacognición, K-BIT y registro de rendimiento académico, todas ellas evaluadas en dos momentos (antes y después del tratamiento). 


\section{Resultados}

Inicialmente se comprobó el grado de homogeneidad de los grupos tratamiento y control en las medidas de ejecución pre-tratamiento. Tal y como se presenta en la Tabla 1, los resultados obtenidos en la $t$-Student, para establecer la diferencia de medias entre grupos ponen de manifiesto que no hay diferencias significativas entre grupos en ninguna de las medidas pre-tratamiento: ni en los subtests de la EHPAP: clasificación $(t(2 / 46)=1.36 ; p \leq$ $.17)$, memoria auditiva $(t(2 / 46)=.546 ; p \leq .58)$, memoria visual $(t(2 / 46)=1.329 ; p \leq .19)$, planificación verbal $(t(2 / 46)=.809 ; p \leq .42)$, series $(t(2 / 46)=.963 ; p \leq .34) \mathrm{y}$ toma de perspectiva $(t(2 / 46)=1.369 ; p \leq .17)$; ni en metacognición $(t(2 / 46)=.112 ; p \leq .91)$, ni en los subtests de vocabulario $(t(2 / 46)=.000 ; p \leq 1)$, matrices $(t(2 / 46)=1.323 ; p \leq .19) \mathrm{y}$ total del K-BIT $(t(2 / 46)=.687 ; p \leq .49)$, ni en rendimiento académico $(t(2 / 46)=.902 ; p \leq .37)$. 
Tabla 1. Diferencia de medias entre grupos en la escala EHPAP, Metacognición, K-BIT y Rendimiento Académico Pre-Tratamiento

\begin{tabular}{|c|c|c|c|c|c|}
\hline & Grupo & Media & D. T. & $t(2 / 46)$ & Sig. \\
\hline \multirow{3}{*}{ EHPAP Clasificación } & Tratamiento & 3.5 & $(1.91)$ & & \\
\hline & Control & 2.79 & (1.66) & 1.36 & .17 \\
\hline & Tratamiento & 2.92 & $(2.46)$ & & \\
\hline \multirow[t]{2}{*}{$\begin{array}{l}\text { EHPAP Memoria } \\
\text { auditiva }\end{array}$} & Control & 3.33 & $(2.80)$ & -.546 & .58 \\
\hline & Tratamiento & 5.21 & $(2.08)$ & & \\
\hline \multirow[t]{2}{*}{$\begin{array}{l}\text { EHPAP Memoria } \\
\text { visual }\end{array}$} & Control & 6.00 & (2.04) & -1.329 & .19 \\
\hline & Tratamiento & 1.29 & (1.94) & & \\
\hline \multirow[t]{2}{*}{$\begin{array}{l}\text { EHPAP Planificación } \\
\text { verbal }\end{array}$} & Control & 1.79 & $(2.32)$ & -.809 & .42 \\
\hline & Tratamiento & 7.54 & $(5.26)$ & & \\
\hline \multirow[t]{2}{*}{ EHPAP Series } & Control & 6.21 & $(4.27)$ & .963 & .34 \\
\hline & Tratamiento & 7.96 & $(3.54)$ & & \\
\hline $\begin{array}{l}\text { EHPAP Toma } \\
\text { perspectiva }\end{array}$ & Control & 9.29 & (3.19) & -1.369 & .17 \\
\hline \multirow{3}{*}{$\begin{array}{l}\text { Metacognición } \\
\text { total pretest }\end{array}$} & Tratamiento & 3.5 & $(2.60)$ & & \\
\hline & Control & 3.58 & $(2.55)$ & -.112 & .91 \\
\hline & Tratamiento & 86.71 & $(16.36)$ & & \\
\hline \multirow[t]{2}{*}{ K.BIT vocabulario } & Control & 86.71 & $(14.26)$ & .000 & 1 \\
\hline & Tratamiento & 106.46 & $(11.02)$ & & \\
\hline \multirow[t]{2}{*}{ K-BIT matrices } & Control & 101.92 & $(12.70)$ & 1.323 & .19 \\
\hline & Tratamiento & 93.75 & (11.93) & & \\
\hline \multirow[t]{2}{*}{ K-BIT total } & Control & 91.21 & $(13.65)$ & .687 & .49 \\
\hline & Tratamiento & 12.64 & $(4.68)$ & .902 & .37 \\
\hline $\begin{array}{l}\text { Rendimiento } \\
\text { académico }\end{array}$ & Control & 11.18 & $(4.27)$ & & \\
\hline
\end{tabular}


Respecto del primer objetivo, consistente en constatar que el grupo tratamiento consigue incrementos significativos del pretest al postest en las tareas de evaluación de dominio, los resultados del análisis de medidas repetidas del modelo lineal general muestran diferencias significativas entre las diferentes puntuaciones pretests y postests en el grupo tratamiento (ver Figura 1) en clasificación $\left(\lambda\right.$ Wilks $\left.(1 / 23)=.316 ; p \leq .001, \eta_{2}=.68\right)$, planificación verbal $\left(\lambda\right.$ Wilks $\left.(1 / 23)=.194 ; p \leq .001, \eta_{2}=.80\right)$, memoria auditiva $(\lambda$ Wilks $\left.(1 / 23)=.495 ; p \leq .001, \eta_{2}=.50\right)$, memoria auditiva recuerdo demorado $(\lambda$ Wilks $(1 / 23=$ $\left..443 ; p \leq .001, \eta_{2}=.55\right)$, vocabulario $\left(\lambda\right.$ Wilks $\left.(1 / 23)=.357 ; p \leq .001, \eta_{2}=.64\right) \mathrm{e}$ interactividad $\left(\lambda\right.$ Wilks $\left.(1 / 23)=.667 ; p \leq .003, \eta_{2}=.33\right)$, con tamaños de efecto medios. No se hallan diferencias significativas en autorregulación.

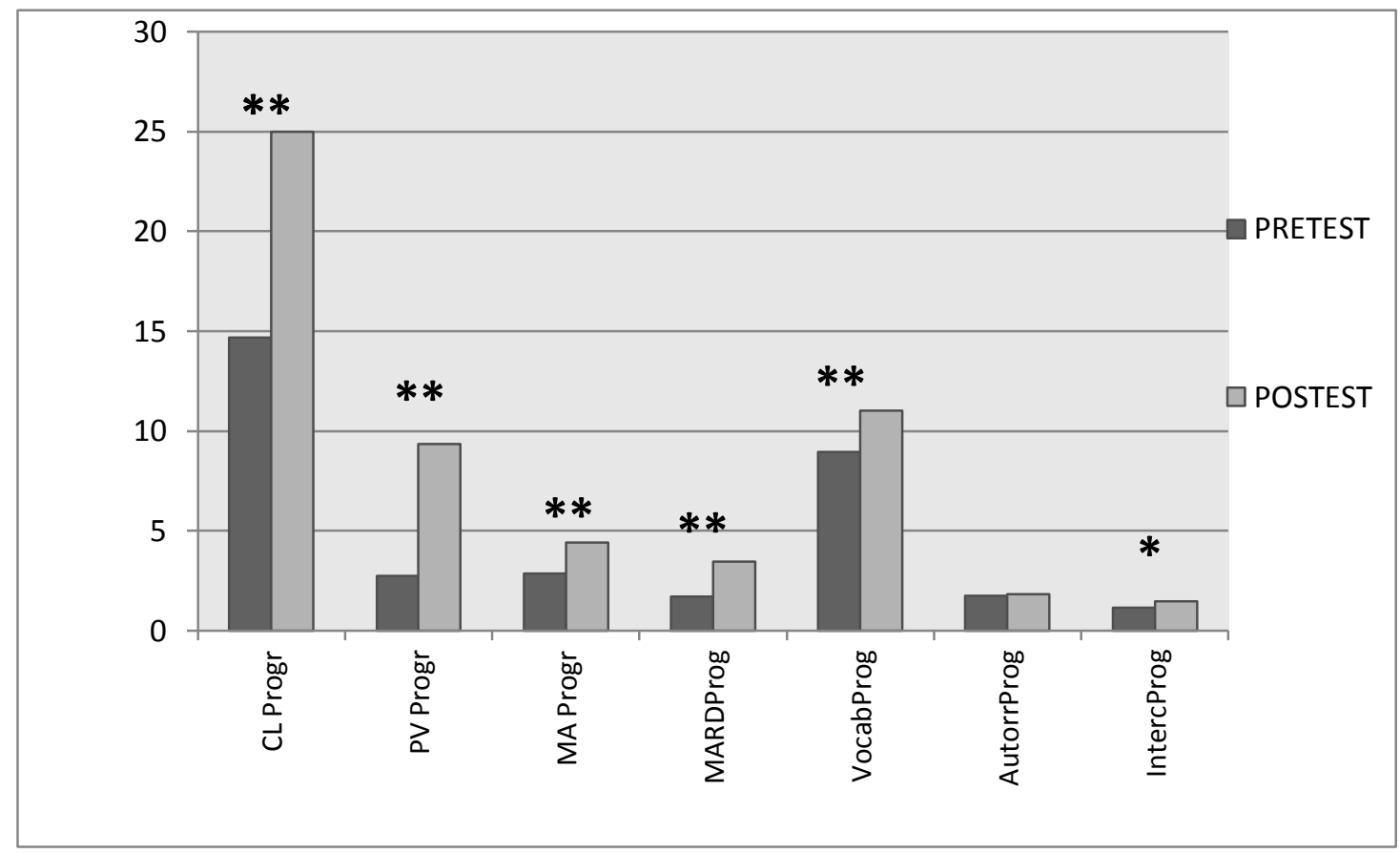

Diferencias significativas entre el pretest y el postest $* p \leq .05, * * p \leq .01$

Figura 1. Medidas repetidas del modelo lineal de las puntuaciones pretests/postests del grupo tratamiento en las tareas de evaluación de dominio próximo a las funciones entrenadas en el programa.

En cuanto al segundo objetivo, destinado a comprobar los efectos diferenciales entre grupo tratamiento y grupo control en la transferencia de efectos del programa a las habilidades cognitivas evaluadas por la escala EHPAP, los resultados del análisis de medidas 
repetidas muestran efecto intragrupo de la interacción entre el factor momento (las dos aplicaciones de las pruebas: pretest/postest) y el factor asistencia al programa (grupo tratamiento/control) en puntuación total $(\lambda$ Wilks $(1 / 47)=.675 ; p \leq .0001)$, planificación $\operatorname{verbal}(\lambda$ Wilks $(1 / 47)=.701 ; p \leq .0001)$, clasificación $(\lambda$ Wilks $(1 / 47)=.867 ; p \leq .011)$, memoria auditiva $(\lambda$ Wilks $(1 / 47)=.886 ; p \leq .019)$ y memoria visual $(\lambda$ Wilks $(1 / 47)=.914$; $p \leq .043)$ de la EHPAP. No se encuentra efecto significativo de la interacción en series y toma de perspectiva (ver Tabla 2).

En estos resultados también se aprecian diferencias significativas intergrupos en el nivel de mejora del pretest al postest en el total de la escala $(F(1 / 47)=18.282 ; p \leq .0001)$, clasificación $(F(1 / 47)=16.740 ; p \leq .0001)$, planificación verbal $(F(1 / 47)=14.255 ; p \leq$ $.0001)$ y series $(F(1 / 47)=6.159 ; p \leq .017)$, a favor del grupo tratamiento. No obstante, las diferencias intergrupos no resultaron significativas en memoria auditiva, memoria visual y toma de perspectiva (ver Tabla 2).

Tabla 2. Modelo lineal de medidas repetidas intragrupo e intergrupos de las puntuaciones pretests/postests para cada subtest de la EHPAP.

\begin{tabular}{|c|c|c|c|c|c|c|c|c|c|}
\hline \multirow[b]{2}{*}{$\begin{array}{l}\text { Subtests } \\
\text { EHPAP }\end{array}$} & \multirow[b]{2}{*}{ Grupos } & \multicolumn{2}{|c|}{ Pretest } & \multicolumn{2}{|c|}{ Postest } & \multicolumn{2}{|c|}{$\begin{array}{l}\text { Intragrupo } \\
\text { interacción }\end{array}$} & \multicolumn{2}{|c|}{ Intergrupos } \\
\hline & & M. & D.T. & M. & D.T. & $\begin{array}{l}\text { Lambda } \\
\text { Wilks }\end{array}$ & $\begin{array}{c}\text { Eta } \\
\text { cuadrado }\end{array}$ & $F(2 / 46)$ & $\begin{array}{c}\text { Eta } \\
\text { cuadrado }\end{array}$ \\
\hline \multirow[t]{2}{*}{ Total } & Tratamiento & 28.42 & (9.69) & 52.50 & $(8.71)$ & $.675 * *$ & .32 & $18.282 * *$ & .28 \\
\hline & Control & 29.42 & (6.67) & 36.79 & $(8.86)$ & & & & \\
\hline \multirow{2}{*}{$\begin{array}{l}\text { Planificación } \\
\text { verbal }\end{array}$} & Tratamiento & 1.29 & (1.94) & 7.13 & (3.79) & $.701 * *$ & .29 & $14.255^{* *}$ & .23 \\
\hline & Control & 1.79 & $(2.32)$ & 2.42 & $(2.82)$ & & & & \\
\hline \multirow[t]{2}{*}{ Clasificación } & Tratamiento & 3.5 & $(1.91)$ & 9.63 & (1.76) & $.867 *$ & .13 & $16.740 * *$ & .26 \\
\hline & Control & 2.79 & (1.66) & 6.46 & (3.38) & & & & \\
\hline \multirow{2}{*}{$\begin{array}{l}\text { Memoria } \\
\text { auditiva }\end{array}$} & Tratamiento & 2.92 & $(2.46)$ & 8.21 & (2.00) & $.886 *$ & .11 & 1.316 & .02 \\
\hline & Control & 3.33 & $(2.80)$ & 6.50 & $(2.53)$ & & & & \\
\hline \multirow{2}{*}{$\begin{array}{l}\text { Memoria } \\
\text { visual }\end{array}$} & Tratamiento & 5.21 & (2.08) & 7.25 & (1.22) & $.914 *$ & .08 & .092 & .00 \\
\hline & Control & 6.00 & $(2.04)$ & 6.67 & $(.91)$ & & & & \\
\hline \multirow[t]{2}{*}{ Series } & Tratamiento & 7.54 & $(5.26)$ & 11.79 & (3.77) & .973 & .02 & $6.159 *$ & .11 \\
\hline & Control & 6.21 & (4.27) & 8.42 & (4.69) & & & & \\
\hline \multirow{2}{*}{$\begin{array}{l}\text { Toma de } \\
\text { perspectiva }\end{array}$} & Tratamiento & 7.96 & $(3.54)$ & 8.50 & (3.51) & .937 & .06 & .008 & .00 \\
\hline & Control & 9.29 & $(3.19)$ & 7.29 & $(3.71)$ & & & & \\
\hline
\end{tabular}

Nota. Existen diferencias significativas entre el pretest y el postest $* p \leq .05,{ }^{*} p \leq .01$ 
En cuanto al tercer objetivo, consistente en comprobar si existen efectos positivos diferenciales entre el grupo tratamiento y el control en el registro de metacognición, el K-BIT y rendimiento académico, los resultados del análisis de medidas repetidas muestran diferencias significativas intragrupo de la interacción entre el factor momento (pretest/postest) y el factor asistencia al programa (tratamiento/control) en metacognición $(\lambda$ Wilks $(1 / 47)=$ $.740 ; p \leq .0001)$. No se observan diferencias significativas en vocabulario, matrices y en C. I. total del K-BIT ni en rendimiento académico.En el análisis intergrupos es también la metacognición $(F(1 / 47)=10.120 ; p \leq .003)$ la única variable que muestra diferencias estadísticamente significativa entre el grupo tratamiento y el control, a favor del primero (ver Tabla 3).

Tabla 3. Modelo lineal de medidas repetidas intragrupo e intergrupos de las puntuaciones pretests/postests para Metacognición, subtests del K-BIT y Rendimiento Académico.

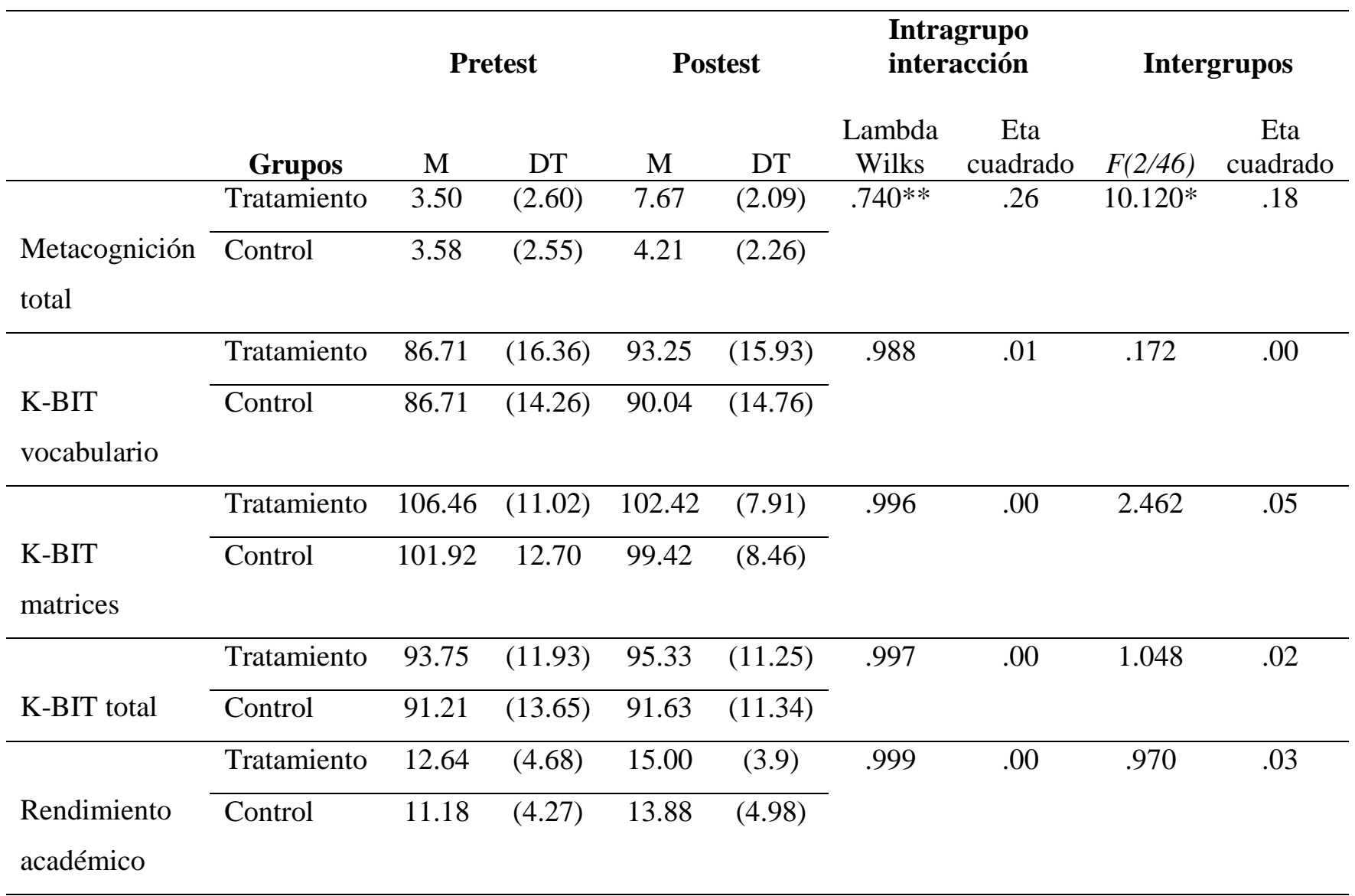

Nota. Existen diferencias significativas entre el pretest y el postest $* p \leq .05, * * p \leq .01$ 


\section{Discusión y conclusiones}

Este estudio se ha dirigido a valorar la eficacia de un programa de entrenamiento cognitivo destinado a preescolares con dificultades en habilidades básicas relacionadas con las competencias exigidas en el currículum de Educación Infantil. Los resultados obtenidos han mostrado que los niños que reciben el programa mejoran significativamente su nivel de ejecución en actividades paralelas al contenido de las sesiones. Así, hemos observado que los niños del grupo tratamiento consiguen aumentar significativamente su habilidad de agrupar en torno a un criterio dado, su memoria auditiva, tanto a corto como a largo plazo, su capacidad de expresar la secuencia de acción de un plan, a la vez que muestran mayor vocabulario e interactuan en mayor medida con el evaluador. La única habilidad evaluada que no muestra diferencias significativas es la de autorregulación. Cabe pensar que esta falta de efecto pueda ser debida a que el programa no se dirige de forma explícita al entrenamiento de esta habilidad, pues, como ya hemos señalado, se ha centrado en tareas próximas al curriculum educativo.

Los resultados obtenidos concuerdan con los hallados en otras investigaciones similares pues, como se revisa en la introducción, generalmente los programas de intervención cognitiva suelen tener efectos positivos en pruebas de dominio diseñadas para su valoración, tanto en grupos de adultos (Calero y García Berben, 1997) como en poblaciones similares a los participantes de este estudio, en concreto el programa FLASH (Gutiérrez, 1999) y el Programa de entrenamiento cognitivo para niños pequeños (Saíz y Román, 2004).

También se ha comprobado que existe una transferencia de los efectos del programa a otras medidas de habilidades cognitivas tales como los subtests de clasificación y planificación verbal de la EHPAP, así como a metacognición, de tal modo que los niños del grupo tratamiento dejan de ser homogéneos frente a los de control. Respecto al resto de las habilidades evaluadas por las EHPAP, aunque sus puntuaciones aumentan del pretest al postest en el grupo tratamiento, y así lo refleja la interación intragrupo momento x asistencia al programa, los efectos no llegan a arrojar diferencias significativas entre grupos para memoria auditiva, memoria visual y toma de perspectiva, problablemente debido al reducido 
número de sujetos que componen la muestra. Del mismo modo, la transferencia no aparece como significativa en los análisis intergrupos ni en inteligencia ni en rendimiento académico.

En definitiva, el Programa de Entrenamiento Cognitivo para Preescolares que valoramos en este trabajo parece mejorar la mayoría de las habilidades sobre las que estaba destinado a actuar. Estos resultados están en la línea de investigaciones previas en las que los programas de entrenamiento se han mostrado lo suficientemente potentes como para generar diferencias de ejecución entre los niños que reciben el programa y los niños del grupo control. Así, los participantes de la mayoría de los programas de entrenamiento cognitivo muestran mejoras en las pruebas de inteligencia general, inteligencia verbal, razonamiento abstracto, rendimiento académico general, materias como lenguaje o matemáticas, habilidades lectoras, funciones metacognitivas, autoconcepto o autoestima, resolución de problemas cognitivos y sociales, etc. (Calero, 1986; Campllonch y cols., 1983; Cerrillo, 2002, 2008; Gutiérrez, 1999, 2002; Lipman, 1989; Narroll y cols., 1982; Pérez y Gutiérrez, 1998; Prieto, 1987; Rand у cols., 1977; Romney y Samuels, 2001; Ruíz Bolívar, 1982; Ruíz Bolívar y Castañeda, 1983; Trickey y Topping, 2004; Saíz y Román, 2004;Yuste, 2007). Debemos destacar la función preventiva que poseen los programas aplicados en esta etapas educativas iniciales, pues éste como el programa FLASH, de Gutiérrez (1999, 2002) o el de Saíz y Román (2004), muestran cómo trabajar con niños de Educación Infantil puede potenciar el desarrollo intelectual del alumno, a la vez que refuerza el desempeño curricular y consigue que los niños mejoren en la aplicación de estrategias de resolución de conflictos cognitivos y sociales.

Es interesante, no obstante, destacar que las diferencias de éste con otros programas al uso le confiere una utilidad educativa que otros programas quizás no posean. La diferencia más notable es que se ha construído para aplicar en pequeño grupo y en sólo 6 sesiones de 45 minutos de duración y con esta intervención tan reducida obtiene efectos similares a programas más amplios o construídos para un uso individualizado.

Estos resultados, junto a los aportados por investigaciones previas, muestran el camino a seguir en los centros educativos como medio para conseguir la potenciación de las capacidades de los menores y la prevención de los futuros problemas académicos que puedan surgir cuando los niños cursan estudios académicos con un mayor grado de exigencia. El objetivo en estas etapas debería ser fortalecer las capacidades cognitivas y metacognitivas 
como vía para reforzar las aptitudes hacia el aprendizaje y para evitar los problemas de aprendizaje y el desajuste escolar.

No obstante, dadas las limitaciones de este trabajo, derivadas del número de sujetos que componen la muestra y su localismo, se hace necesario replicar esta investigación y complementarla con un seguimiento de los niños participantes en el estudio, con el objeto de determinar la durabilidad de los efectos.

\section{Agradecimientos}

Este trabajo forma parte del Proyecto de Excelencia de la Junta de Andalucía "Perfiles de aprendizaje y pautas de crianza en preescolares inmigrantes: un abordaje integral para la prevención de problemas educativos de rendimiento y convivencia. REF. P07HUM2535". También, del Programa de Formación de Profesorado Universitario (FPU). Ministerio de Educación. Gobierno de España.

\section{Referencias}

Annevirta, T. y Vauras, M. (2006). Developmental changes of metacognitive skill in elementary school children. Journal of Experimental Education, 74, 197-225.

Bensoussan, Y. (2002). The effectiveness of mediation on three subtests of the aplication of cognitive functions scale, a dynamic assessment procedure for young children. (Unpublished Master's thesis). New York: Touro Collage.

Calero, M. D. (1986). Valoración del programa de Enriquecimiento Instrumental en una muestra de adolescentes andaluces. Siglo Cero (julio-agosto), 50-56.

Calero (Coord.) (1995). Modificación de la inteligencia: sistemas de evaluación e intervención. Madrid: Pirámide.

Calero, M. D. y García-Berbén, T. M. (1997). A self-training program in inductive reasoning for low-education elderly: tutor-guided training vs. self-training. Archives of Gerontology and Geriatric, 24, 249- 259.

Calero, M. D., Carles, R., Mata, S. y Navarro, E. (2010a). Diferencias en habilidades y conducta entre grupos de preescolares de alto y bajo rendimiento escolar. Revista Electrónica de Investigación y Evaluación Educativa, R.E.L.I.E.V.E., 16 (2). Recuperado de http://www.uv.es/RELIEVE/v16n2/RELIEVEv16n2_5.htm

Calero, M. D., Mata, S. y Carles, R. (2011). PROMECO: Programa Mediacional de Enriquecimiento Cognitivo para niños de Educación Infantil. Madrid: EOS. 
Calero, M. D., Robles, M. A y García-Martín, B. (2010b). Habilidades cognitivas, conducta y potencial de aprendizaje en preescolares con Síndrome de Down. Electronic Journal of Research in Educational Psychology, 8 (1), 87-110.

Calero, M., Robles, M., Márquez, J. y De la Osa, P. (2009). EHPAP: Evaluación de habilidades y potencial de aprendizaje en preescolares. Madrid: EOS.

Campllonch, J. M., Domínguez, M. T., Beardo, J., Matías, A. y Navarro, J. (1983). Aplicación de un Programa de Enriquecimiento Instrumental para alumnos de EGB. Cádiz: ICE Universidad de Cádiz.

Carles, R. (2012). Potencial de aprendizaje y adaptación al contexto educativo. La prevención de problemas escolares en niños inmigrantes (Tesis Doctoral no publicada). Universidad de Granada, Granada.

Carles, R., Mata, S., López-Rubio, S., Navarro, E., Calero, M. D., Fernández-Parra, A., Vives, C. y Márquez, J. (2010). Perfil de Aprendizaje y Potencial de Aprendizaje en Preescolares Inmigrantes y Españoles. En Soriano, E., González, A. y Zapata, R. Retos Internacionales ante la Interculturalidad (pp. 504-512). Almería: Editorial de la Universidad de Almería.

Castañón, N. (2007). Efecto diferencial del programa Bright Start en sujetos venezolanos de estrato social medio y bajo. Equisangulo. Revista Iberoamericana de Educación Matemática, 4 (2). Recuperado de

http://www.saber.ula.ve/dspace/bitstream/123456789/20327/1/articulo3.html

Cerrillo, R. (2002). Enseñar a pensar: desarrollo del razonamiento lógico. Aula Abierta, 80, 63-83.

Cerrillo, R. (2008). Programa para enseñar a pensar CORAL. Guía didáctica y solucionarios. Madrid: CEPE.

De la Cruz, V. (1982). Batería de Aptitudes para el Aprendizaje, BAPAE. Madrid: TEA.

Desoete, A. (2007). La evaluación y mejora del proceso de enseñamnza-aprendizaje de las matemáticas a través de la metacognición. Electronic Journal of Research in Educational Psychology, 13 (5), 705-730.

Domínguez, Mallon, Warnick, Nickenson, Sánchez y cols. (1980). Proyecto de Inteligencia Hardvard, Venezuela, Ministerio para el Desarrollo de la Inteligencia.

Edfeldt, A. W. (1955); Villega, M. (1986). Reversal Test. Barcelona. Herder.

Feuerstein, R. (1993). La teoría de la modificabilidad estructural cognitiva: un modelo de evaluación y tratamiento de los procesos de la inteligencia. En Beltrán, J. Bermejo, V.; 
Prieto, Ma. D. y Vence, D. (Eds.). Intervención psicopedagógica (pp. 5-27). Madrid: Pirámide.

Feuerstein, R., Rand, Y., Hoffman, M. B. y Miller, R. (1980). Instrumental Enrichment: an intervention program for cognitive modifiability. Baltimore: University Park Press.

Feuerstein, R., Klein, P., y Tannenbaum, A. (1991). Mediated Learning Experience (MLE): Theoretical Psychosocial and Learning Implications. England: Freund Publishing House Ltd.

Feuerstein R, Feuerstein R. S., Falik, L. H. y Rand, Y. (2002). The dynamic assessment of cognitive modifiability: The learning propensity assessment device: theory, instruments and techniques. Israel: The ICELP Press.

Francesc, M., Serrano, J. y Carles, A. (2010). Mejora de la comprensión social de niños preescolares: Evaluación de un programa de formación. Electronic Journal of Research in Educational Psychology, 8 (2), 841-860.

Garrett, A. J., Mazzocco, M. M. y Baker, L. (2006). Development of the metacognitive skills of prediction and evaluation in children with or without math disability. Learning Disabilities Research and Practice, 21(2), 77-88.

Gutiérrez, J. (1999). Programa FLASH. Madrid: III Congreso Internacional de Psicología y Educación. Distribuidora SEK.

Gutiérrez, J. (2002). Entrenamiento cognitivo en el primer ciclo de la educación primaria. Tesis Doctoral. Universidad Complutense, Madrid.

Haywood, H. (2000). Bright Start: cognitive curriculum for young children. L'éducation et remédiation cognitive. 25 Octubre, Conférence.

Haywood, H., Brooks, P. y Burns, S. (1986). Stimulating cognitive development at developmental level: A tested non-remedial preschool curriculum for preschoolers and older retarded children. Special Services in the Schools, 3 (1), 127-147.

Haywood, H., Brooks, P. y Bums, S. (1992). Bright Start: Cognitive Curriculum for Young Children.Watertown, MA: Charlesbridge Publishing.

Hunt, J. (1961). Inteligencia y Experiencia. New York: Roland.

Justicia, F., Amézcua, J. A., y Pichardo, M. C. (Coords.) (2000). Programas de intervención cognitiva. Granada: Grupo Editorial Universitario.

Kaufman, A. y Kaufman, A. (1994). K- BIT: Test Breve de Inteligencia de Kaufman. Manual de interpretación. Madrid: TEA.

Lidz, C. S. (2004). Assessment procedure with deaf student between the ages of for and eight years. Educational and Child Psychology, 21(1), 59-73. 
Lidz, C. S. y Van der Aalsvoort, G.M. (2005). Usefulness of the Application of Cognitive Functions Scale with young children from the Netherlands. Transylvanian Journal of Psychology, 1, 82-99.

Lipman, M. (1989). Pixie. Madrid: Ediciones de la Torre.

Malowitsky, M. (2001). Investigation of the effectiveness of the mediation portion of two subtests of the Application of Cognitive Function Scale, a dynamic assessment procedure for young children (Unpublisher Master's thesis). New York: Touro College.

Marcel, V., Bernadette H.A.M. y Afflerbach, P. (2006). Metacognition and learning: conceptual and methodological considerations. Metacognition Learning, 1 (1), 3-14.

Meilán, J., Salgado, V., Arana, J., Carro, J. y Jenaro, C. (2008). Entrenamiento cognitivo y mejora de la memoria prospectiva en jóvenes con retraso mental leve. Revista de Investigación Educativa, $26 \quad$ (1), 227-245. Recuperado de http://revistas.um.es/rie/article/view/99351

Monereo, C. (1994). Estrategias de enseñanza y aprendizaje. Barcelona: GAO.

Narrol, H., Silverman, H. y Waksman, M. (1982). Developing cognitive potential in vocational high school student. Journal of Educational Research, 76 (2), 107-112.

Pelechano, V. (1984). Inteligencia social y habilidades interpersonales. Análisis y Modificación de Conducta, 10, 394-421.

Pérez, L. y Gutiérrez, P.J. (1998). Programa FLASH. Refuerzo del desarrollo cognitivo. Manual del profesor. Madrid: CEPE.

Prieto, M. D. (1987). El potencial de aprendizaje: Un modelo y un sistema aplicado de evaluación. Murcia: ICE.

Rand, Y., Feuerstein, R., Tannenbaum, A., Jesen, M. y Hoffman, M. (1977). An analysis of effects of Instrumental Enrichment on Disadvantaged adolescents. En Mittler P. (Eds.). Research to practice in mental retardation: Education and training (Vol. 2). Baltimore, MD: University Park Press.

Raven, J. C. (1996). Raven: matrices progresivas: CPM, SPM, APM. Madrid: TEA.

Romney, D. y Samuels, M. (2001). A meta-analytic evaluation of Feuerstein's Instrumental Enrichment program. Educational and Child Psychology, 18(4), 19-34.

Ruiz Bolívar, C. (1988). Efecto del programa enriquecimiento instrumental en la modificabilidad cognitiva de estudiantes pre-universitarios. AVIED, 3, 33-44.

Ruíz Bolívar, C. y Castañeda, E. (1983). Efectos del Programa de Enriquecimiento Instrumental sobre factores cognoscitivos y no cognoscitivos en sujetos de diferentes estratos socioeconómicos. Venezuela: Universidad Nacional Experimental de Guayana. 
Saíz, M.C. (1995a). Adaptación y validación de un Programa de Desarrollo Socio-cognitivo en Niños con Deprivación Socio-ambiental (Tesis Doctoral no publicada). Universidad de Valladolid, Valladolid.

Saíz, M.C. y Román, J.M. (2004). Programa de entrenamiento cognitivo para niños pequeños. Madrid: CEPE.

Soriano, E. y Franco, C. (2010). Mejora de la autoestima y de la competencia emocional en adolescentes inmigrantes sudamericanos residentes en España a través de un programa psicoeducativo de mindfulness (conciencia plena). Revista de Investigación Educativa, 28 (2), 297-312. Recuperado de http://revistas.um.es/rie/article/view/94191

Trickey, S. y Topping, K. J. (2004). Philosophy for children: a systematic review. Research Papers in Education, 19(3), 365 - 380.

Tzuriel, E., Kaniel, S., Kaner, A. y Haywood, H. C. (1997). The effectiveness of Bright Start program in kindergarten on transfer abilities and academic assessment. Early Child Development Quarterly.

Tzuriel, D., Kaniel, S., Zeliger, M., Friedman, A. y Haywood, H. (1998). Effects of the "Bright Start" Program in Kindergarten on Teachers. Use of Mediation and Children's Cognitive Modifiability, Early Child Development and Care, 143 (1), 1-20.

Vanden Wijngaert (1991). Cognitive education with speech and language disordered children. The Thinking Teacher, 6, 1-5.

Warnez, J. (1991). Implementation of the CCYC in a therapeutic center. The Thinking Teacher, 6, 7-9.

Yuste C. y Sánchez, J. (2007). Colección Progresint: Programa para la estimulación de las habilidades de la inteligencia. PROGRESINT Manual. Madrid: CEPE. 